Revue d'histoire de l'Amérique française

TEVVUE D.HISTOIRE DE L'AMÉRIQUE FRANÇAISE

\title{
L'utilité du concept de mode de production des petits producteurs pour l'historiographie de la Nouvelle-France
}

Denis Monière

Volume 29, numéro 4, mars 1976

URI : https://id.erudit.org/iderudit/303482ar

DOI : https://doi.org/10.7202/303482ar

Aller au sommaire du numéro

Éditeur(s)

Institut d'histoire de l'Amérique française

ISSN

0035-2357 (imprimé)

1492-1383 (numérique)

Découvrir la revue

Citer cet article

Monière, D. (1976). L'utilité du concept de mode de production des petits producteurs pour l'historiographie de la Nouvelle-France. Revue d'histoire de l'Amérique française, 29(4), 483-502. https://doi.org/10.7202/303482ar d'utilisation que vous pouvez consulter en ligne. 


\title{
L'UTILITÉ DU CONCEPT DE MODE DE PRODUCTION DES PETITS PRODUCTEURS POUR L'HISTORIOGRAPHIE DE LA NOUVELLE-FRANCE
}

\author{
DENIS MONIÈRE \\ Département de science politique \\ Université d'Ottawa
}

Lorsqu'on relit la littérature analytique portant sur la société de la Nouvelle-France, on constate qu'historiens et sociologues décrivent la plupart du temps les structures sociales (régime seigneurial), le système politique (l'absolutisme), les activités économiques (traite des fourrures et agriculture), en réduisant la complexité de cette formation sociale soit au féodalisme pour l'école de Québec, soit à l'émergence d'une structure économique de type capitaliste pour l'école de Montréal. Ces deux orientations historiographiques prennent pour acquis l'existence en métropole d'une structure sociale relativement simple: une société féodale en décomposition sous la pression du développement du capitalisme.

Il nous semble que d'une part on a simplifié la structure économique métropolitaine et d'autre part on a fait l'économie d'une véritable étude du mode de production et des forces productives de la colonie en procédant par analogie ${ }^{1}$. Nous pensons que l'application à l'étude de la Nouvelle-France du concept de mode de production des petits producteurs ${ }^{2}$ (MPPP) pourrait être utile et éclairer de façon plus pertinente les particularités de l'économie politique de la

1 D'ailleurs, jusqu'à présent, l'historiographie québécoise a privilégié l'instance politique comme lieu principal des contradictions, facteur décisif de l'explication historique et instrument de césure diachronique de sorte que l'histoire du Québec a été celle de ses régimes politiques et non de ses modes de production. Il faut toutefois souligner que depuis quelques années de plus en plus d'historiens s'éloignent de cette orientation et mettent l'accent sur les conditions matérielles de l'existence. La recherche de Louise Dechêne, Habitants et marchands de Montréal au XVII e siècle (Paris, Plon, 1974) illustre bien cette nouvelle tendance.

2 Dans la suite du texte, nous utiliserons le sigle MPPP pour désigner le mode de production des petits producteurs.

RHAF, vol. 29, no 4 (mars 1976) 
Nouvelle-France. Ce concept pourrait renouveler notre compréhension de la formation sociale québécoise et du développement du capitalisme. Nous nous proposons ici de reposer brièvement la problématique de la transition, de discerner la spécificité du MPPP, de voir en quoi il peut correspondre à la situation de la Nouvelle-France et enfin comment cette nouvelle perspective affecte l'interprétation traditionnelle de la formation des classes au Québec. Nous ne prétendons pas dépasser le stade de l'hypothèse exploratoire. Nous nous contenterons de présenter quelques critères théoriques qui pourraient, dans des recherches ultérieures, nous permettre d'identifier ce mode de production particulier et de souligner quelques illustrations empiriques de sa pertinence. Puisque, pour ce faire, nous utiliserons les résultats d'études basées sur des problématiques divergentes, nous ne pouvons nous attendre à une adéquation parfaite entre les faits observés et le modèle théorique que nous présentons. Ce n'est qu'à titre indicatif et non pas démonstratif que nous nous référons aux travaux existants.

\section{Poser le problème de la transition...}

La méconnaissance d'une problématique de la transition, outre qu'elle interdit de penser théoriquement un certain nombre de décalages entre l'infrastructure et la superstructure, comme à certaines périodes le primat du politique et des formes d'intervention propres au politique, entraine des contresens et des faux problèmes. ${ }^{3}$

Ce reproche pourrait être appliqué à l'historiographie du régime français et du régime anglais au Canada. La plupart de nos historiens se réfèrent à des modèles abstraits, s'enferment dans une logique formelle de la succession des modes de production et de la sorte escamotent trop souvent la complexité du réel et la spécificité du concret. Les décalages, retards, asymétries semblent les gêner et leur être intolérables. Certains veulent établir la dominance du capitalisme sous le régime français alors que pour d'autres ce même régime appartient à l'âge féodal et réactionnaire, le capitalisme ne se développant qu'après la Conquête, sous l'influence bénéfique des marchands anglais, principaux artisans des progrès du Canada.

Les modes de production ne se détruisent pas mécaniquement et ne se succèdent pas à la queue leu leu, à la manière des wagons

3 Régine Robin «La nature de l'État à la fin de l'Ancien régime», Dialectiques, nos 1-2 (1973): 42. 
de chemin de fer. Ils s'interpénètrent plutôt dans des rapports dialectiques de continuité, de rupture, de recul, d'équilibre et de déséquilibre. Les formes capitalistes dans leur développement ne sont donc pas pures. Pour les comprendre, il faut les saisir dans le cadre de la transition où elles disloquent, adaptent et parfois maintiennent les anciennes formes de rapports de production. Les phases de développement d'une formation économico-sociale ne se tranchent pas au couteau. Le processus d'établissement d'une dominance est long et marqué de développements inégaux, ce qui suppose la possibilité de cœxistence de plusieurs modes de production. Comprendre la transition implique la compréhension de l'articulation des modes de production, ce qui n'est possible qu'en abandonnant la problématique de l'unité ou de l'homogénéité en ce qui concerne la façon de produire l'existence. Ce n'est pas parce que dans une formation sociale à un moment donné il y a absence d'un Etat libéral, d'une bourgeoisie constituée selon des critères formels ou encore d'une éthique capitaliste qu'il n'y a pas dominance du mode de production capitaliste, à moins de postuler une adéquation parfaite entre l'instance économique et les superstructures politiques et idéologiques, ce qui semble peu probable sur le plan historique.

Dans cet ordre d'idées, on peut considérer, comme le fait Poulantzas, qu'il y a autonomie relative de l'État sous la monarchie française du XVIIe siècle:

La grande majorité des historiens sont d'accord sur le fait que la coupure entre l'état féodal et l'état capitaliste n'a pas lieu au moment où apparait l'État correspondant à la consolidation de la domination politique de la classe bourgeoise dont l'État issu de la Révolution française offrirait l'exemple caractéristique, mais bel et bien au moment où apparaît l'État absolutiste. ${ }^{4}$

De la même façon, on peut penser la bourgeoisie comme étant intégrée au système féodal et pas toujours en contradiction antagonique avec la noblesse:

Car la bourgeoisie d'ancien régime est d'une nature complexe, écrit Claude Mazauric. En accumulant le capital, elle devient accapareuse de rente foncière, créancière de l'Etat, profiteuse de colonies et surtout ambitieuse de pénétrer dans l'appareil d'État par l'acquisition d'offices et dans la noblesse par l'achat de magistratures ou de lettres patentes; longtemps elle collabore 168-169.

N. Poulantzas, Pouvoir politique et classes sociales (Paris, Maspéro, 1968), 
avec la noblesse sous la houlette du roi-arbitre. Comme telle, cette bourgeoisie s'installe dans le régime féodal autant qu'elle le mine. ${ }^{5}$

Donc, dans un stade préliminaire, la bourgeoisie a besoin de l'ordre féodal pour croître. Dès lors, l'existence d'une bourgeoisie forte et même dominante n'est pas incompatible avec le maintien momentané de structures sociales féodalistes. C'est d'ailleurs ce qui se produit en Angleterre au XVIII ${ }^{\mathrm{e}}$ siècle où s'opère la symbiose entre l'aristocratie foncière et la bourgeoisie.

Si sur le plan heuristique, il est nécessaire pour comprendre et expliquer le développement des formations sociales de les penser à travers la problématique de la transition, de la cœxistence des modes de production et de dégager les rapports de force entre classe dominante et classes dominées, cela ne signifie pas qu'il faille pour autant, dans cette démarche, procéder selon une logique binaire. La lutte ne se fait pas toujours à deux. Féodalisme et capitalisme n'occupent pas nécessairement tout le champ de la réalité sociale.

\section{Un troisième larron: le MPPP}

C'est Marx qui a pressenti la possibilité de la présence d'une troisième composante, c'est-à-dire un mode de production obscur, passé inaperçu qui fait généralement figure de victime de l'histoire. Marx en parle à quelques reprises mais sans lui donner de nom précis. C'est en quelque sorte le caméléon de l'histoire. La difficulté principale présentée par ce curieux mode de production est la facilité avec laquelle il s'associe avec d'autres modes de production, par exemple, le capitalisme. Avec celui-ci la personnalité du petit producteur se dédouble, il est à la fois son propre capitaliste et son propre salarié, il se transforme aisément en petit capitaliste. "Nous rencontrons là, dit Marx, une particularité qui caractérise une société dans laquelle prédomine un mode de production déterminé, bien que tous les rapports de production n'y soient pas encore assujettis.» 6

Guy Dhoquois a cherché à approfondir cette particularité et décrit en ces termes les principales caractéristiques de ce mode de production:

Le mode de production des petits producteurs consiste dans des

5 Cl. Mazauric, Sur la révolution française (Paris, Ed. Sociales, 1970), 93.

6 K. Marx, Oeuvres: matériaux pour l'économie (Pléiade), I: 401 ss. 
travailleurs qui sont en même temps propriétaires à titre individuel de leurs moyens de production et qui n'exploitent pas ou guère le travail d'autrui. Il aboutit dans bien des cas à une petite économie marchande... Dans ce mode de production, il n'y a même pas à proprement parler de classes sociales puisqu'il n'y a pas de classes antagonistes... Mais Marx a rappelé à son sujet un mot terrible. Son absence même d'antagonisme social le condamnait à la «médiocrité». Sans cesse, il était recouvert par d'autres modes de production, à classes antagonistes ceux-là, plus dynamiques, plus agressifs. ${ }^{7}$

S'il ne peut être dominant, il ne peut non plus être éliminé car il se reconstitue sans cesse à travers les multiples transformations qui s'opèrent dans les formations sociales, de sorte qu'on le retrouve dans le mode de production esclavagiste, féodal et capitaliste. S'il est «médiocre», il n'est pas pour autant insignifiant, c'est-à-dire qu'il peut s'insérer dans le processus historique et y jouer un rôle actif qui pourrait à la limite expliquer l'asymétrie dans le développement des composantes de la totalité sociale.

Dans ce type d'économie prédominent les aspects suivants: le travailleur est propriétaire de ses instruments de production, il produit tout ce qui est nécessaire à sa reproduction et à la reproduction sociale. Les producteurs sont sur un pied d'égalité les uns par rapport aux autres. Il y a quasi-autarcie ou autosuffisance. Il peut y avoir production de plus-value, mais il n'y a pas d'extorsion directe de plus-value. L'échange, lorsqu'il y en a, ne prend pas la forme marchande mais plutôt la forme simple. La production n'est pas faite pour le marché, il y a donc peu d'accumulation du capital. Enfin ce mode de production ne peut avoir de superstructure qui lui soit propre. L'agent économique propre à ce mode de production est l'artisan et le paysan. Selon Guy Dhoquois, le MPPP se serait développé en France à la suite du processus de décomposition du féodalisme et en ce sens serait une conséquence inattendue de développement du capitalisme. Et contrairement à ce qu'on peut penser, il fut renforcé par la Révolution française qui sanctionna au niveau juridique la petite propriété parcellaire.

Mais il faut ici aller au-delà de la simple énumération de critères et décrire les principaux aspects qui délimitent la particularité de ce mode de production au niveau des relations marchandes, des relations sociales de production, des relations techniques de production et enfin des relations avec le mode de production dominant.

7 Guy Dhoquois, Pour l'histoire (Paris, Anthropos, 1971), 189-190. 
Dans une société rurale, on peut distinguer différents types de production. Il existe la production de biens pour la consommation personnelle et la production artisanale d'articles commandés par d'autres paysans pour leur propre consommation. Ce type de marché n'est pas capitaliste en ce sens que le marché n'est pas généralisé, c'est-à-dire que les produits ne sont pas faits pour des consommateurs inconnus et indifférenciés. Ces artisans ou ces petits producteurs, selon le terme de Lénine ${ }^{8}$, ont des contacts directs avec le marché, ils produisent des articles qui leurs sont commandés directement par le client pour sa propre utilisation. Le principal résultat de leur activité productive est simplement la reproduction de leur moyen de subsistance. Ils ne sont pas soumis au processus de l'aliénation marchande même s'ils produisent une valeur d'échange. Ils ont un rapport personnel au produit qu'ils créent. Ils se préoccupent de la qualité du produit et tirent une satisfaction personnelle de leur travail. C'est principalement la valeur d'usage qui détermine l'activité du producteur.

On peut retrouver surtout dans les viiles une forme pius avancée de MPPP, c'est-à-dire plus articulée au mode de production capitaliste: la production marchande simple, qui existe lorsque le petit producteur engage des travailleurs qui produisent une plus-value et permettent ainsi une accumulation de capital. Cette accumulation se traduit alors par l'élargissement de l'unité de production à la fois en termes de quantité de travail utilisé et de moyens de production employés. À ce niveau de développement, la valeur d'échange prédomine sur la valeur d'usage car l'intérêt principal de l'activité productive est la réalisation du profit et non plus le produit lui-même. Il y a distanciation dans le rapport producteur-consommateurs.

En ce qui concerne les relations sociales de production du MPPP son aspect principal est une relation de dépendance personnelle de type paternaliste, en ce sens où l'apprenti dépend du maître-artisan pour le logement, la nourriture et l'habillement. Dans sa forme artisanale, l'apprenti est habituellement un membre de la famille à qui le père transmet ses connaissances et ses moyens de production. Dans sa forme marchande simple, les apprentis n'ont pas la plupart du temps de liens familiaux avec le maître-artisan, ils sont plus nombreux et passent plus rapidement au statut de journaliers. L'apprenti ici n'est pas encore à proprement parler un salarié,

8 Lénine, Le développement du capitalisme en Russie (Moscou, Éditions du Progrès, 1974). 
car il reçoit une part des profits et peut à l'occasion (dans les temps morts) utiliser les moyens de production à son propre compte. Ayant acquis la compétence nécessaire, il espère d'ailleurs pouvoir un jour être indépendant et s'établir à son propre compte.

En ce qui concerne les relations techniques de production, le petit producteur effectue l'ensemble des opérations de production. Il choisit la matière première (il arrive qu'il la produise lui-même), il contrôle le procès de production décidant quand et comment il travaillera, quels outils il emploiera et comment il les utilisera. Il n'a besoin de personne pour lui dire que faire ni comment le faire. Dans la phase artisanale, la division du travail est faible, dans la phase marchande simple elle tend à s'accroître et à fonder des rapports de subordination.

En Europe, dans la période de transition opérée par la révolution industrielle, le développement du capitalisme a entrainé la dissolution du MPPP, la majorité des petits producteurs devenant soit des petits capitalistes, soit des prolétaires. Cette dissolution a toutefois permis la conservation d'un secteur artisanal résiduel limité à certaines activités bien spécifiques.

$\mathrm{Au}$ contraire, en situation coloniale ou dans les économies extraverties, on remarque que dans l'articulation au mode de production dominant, c'est l'aspect conservation et non la dissolution qui prédomine ${ }^{9}$. En général, les secteurs à faible rentabilité comme ceux des biens de consommation et des services sont laissés aux petits producteurs indépendants, ce qui permet aux capitalistes d'avoir les coudées franches pour maximiser les profits dans les secteurs les plus rentables. Mais de toutes façons, si le mode de production capitaliste ne détruit pas le MPPP, il se le subordonne. L'effet de cette conservation est de retarder le procès de prolétarisation, d'assurer un rôle social privilégié à la petite bourgeoisie, de structurer la conscience autour de l'attachement à la famille, à la religion et aux liens ethniques et aussi d'entraver le développement d'une conscience de classe.

\section{Le MPPP et la formation économico-sociale québécoise}

À notre avis, l'utilisation de ce concept pour l'étude de la formation sociale québécoise nous donnerait une vue plus juste de no-

9 Voir Olivier Lebrun et Chris Gerry, «Petty Producers and Capitalism», $R e$ view of African Political Economy, no 3 (mai-octobre 1975): 29-30. 
tre développement historique et permettrait de reviser certaines thèses. Pour l'instant, seuls à notre connaissance, Marcel Rioux et ses collaborateurs ont envisagé cette hypothèse, limitant son application cependant à l'après-Conquête jusqu'à la Confédération :

les Québécois restent en dehors du système socio-économique des colonisateurs anglais, ils participent - dans les régions rurales où ils habitent en grande majorité - à un mode de production qui n'est ni le mode féodal, ni le mode capitaliste. Qu'est ce à dire? Au Québec, s'est développé un mode de production transitoire qui à cause de l'antagonisme dominé-dominant, colonisateur-colonisé, s'est maintenu pendant plusieurs décennies et a profondément influencé cette formation sociale. C'est un mode de production que Guy Dhoquois propose d'appeler "mode de production des petits producteurs". ${ }^{10}$

Malheureusement, les auteurs ne font pas travailler outre mesure cet instrument théorique et nous laissent sur une piste de recherche qui tout en étant prometteuse n'est pas encore débroussaillée. On perçoit hien aussi que la problématique des effets de la Conquête est sous-jacente à cette hypothèse.

Pour notre part, nous aurions tendance à penser que le MPPP n'est pas particulier au régime anglais mais qu'il prend plutôt racine sous le régime français en tant que mode de production sous-jacent au développement du capitalisme dans sa phase commerciale et que l'effet spécifique de la Conquête, quant à l'articulation des modes de production, a été de généraliser le MPPP et d'établir sa dominance à la base de la structure sociale canadienne-française.

En ce sens, nous pensons que la thèse de Gilles Bourque qui confine aux superstructures les effets de la Conquête devrait être révisée pour tenir compte de cette modification de la base économique de la structure sociale canadienne-française résultant du changement de métropole. S'il est possible de penser l'autonomie des superstructures, il n'est pas impossible d'envisager sur le plan théorique un effet positif de cette autonomie sur l'infrastructure elle-même. Il s'agirait donc de relativiser la thèse de l'absence de changements dans la structure économique interne de la colonie et dans cette perspective, la Conquête aurait eu pour effet de renforcer un mode de production présent mais non dominant dans la formation sociale de la Nouvelle-France. Évidemment, cette hypothèse n'est soutenable que

10 Y. Lamarche, M. Rioux et R. Sévigny, Aliénation et idéologie dans la vie quotidienne des Montréalais francophones (Montréal, P.U.M., 1974), 32. 
si on se dégage de la perspective mécaniste qui met en opposition le féodalisme et le capitalisme.

Il va de soi aussi que cette hypothèse implique une réinterprétation des fondements économiques de la Nouvelle-France qui, tout en étant en transition vers le capitalisme, se caractérise par la combinaison du MPPP à pellicule féodaliste et du capitalisme commercial. Cette interprétation a déjà été proposée et soutenue par Larry MacDonald selon qui: «the history of Quebec, until Confederation at least, seems to belong, structurally, to the more universal category of a petty bourgeois economy hosting emergent capitalist profiles.» ${ }^{11}$

Nous essaierons maintenant de présenter quelques observations qui tendent à soutenir la plausibilité de cette hypothèse.

Jusqu'à présent, nos historiens se sont braqués sur les aspects superstructuraux pour comprendre la nature de la formation sociale de la Nouvelle-France et, dans cette perspective, le débat a consisté à déterminer quelle était l'élite ou la classe dirigeante de la Nouvelle-France. D'un côté, on retrouve ceux qui soutiennent que la Nouvelle-France était une société dynamique dominée par une élite «bourgeoise" à composition hétéroclite (C. Nish) et de l'autre ceux qui affirment au contraire que c'était une société dominée par la noblesse réactionnaire et un État absolutiste. On a donc cherché à savoir qui composait l'élite et quelles étaient ses caractéristiques en négligeant la base de la formation des classes, c'est-à-dire les rapports de production.

L'argumentation de Jean Hamelin est significative à cet égard car pour lui, l'absence d'une bourgeoisie coloniale est attestée par l'état de développement économique de la colonie, ce qui signifie dans sa perspective insuffisance d'immigrants spécialisés et cossus, difficultés de constituer une main-d'oeuvre spécialisée autochtone, structure commerciale qui favorise l'enrichissement métropolitain au détriment de la bourgeoisie coloniale, incapacité d'effectuer des transferts de capitaux dans d'autres secteurs économiques et de diversifier l'économie ${ }^{12}$. On a l'impression que la démarche logique de

11 Larry MacDonald, «Petty Bourgeois Aspects of Pre-Industrial Canada» (texte multigraphié), 15. Voir aussi «France and New France: the Internal Contradictions ", Canadian Historical Review, LII, no 2 (juin 1971): 121-143.

12 Jean Hamelin, Économie et société en Nouvelle-France (Québec, P.U.L. 1960), 135-136. Voir aussi Fernand Ouellet, Histoire économique et sociale du Québec (Montréal, Fides, 1966), 5. 
ces historiens consiste à chercher à l'origine les résultats du processus de développement lui-même, c'est-à-dire de prendre les caractéristiques du capitalisme constitué et dominant et de postuler la nécessité de leur existence à l'origine du processus. De même, selon F. Ouellet, pour qu'il y ait capitalisme et bourgeoisie, il faut qu'il y ait en plus une mentalité, un mode de pensée propre au bourgeois, une idéologie libérale. Ces auteurs pratiquent le téléscopage du processus historique. F. Ouellet et J. Hamelin, dans leur démarche analytique, marchent sur la tête. Ils expliquent les classes par les idées qu'elles se font d'elles-mêmes, au lieu de considérer cette question en partant de la place occupée dans le procès de production.

L'analyse de Cameron Nish, pour pertinente qu'elle soit, n'est pas tout à fait dégagée de cette logique vicieuse et élude comme les autres l'analyse des rapports sociaux de production. Il adopte une perspective fonctionnaliste visant à démontrer que de 1729 à 1748 on rencontre en Nouvelle-France des occupations relevant des fonctions de la classe bourgeoise ${ }^{13}$. Il constate que la distinction traditionnelle nobblesse-bourigeoisie ne colle pas à la réalité de la Nouvelle-France où pour ainsi dire on retrouve un bloc historique composite :

Nous pouvons donc conclure, que l'administration militaire, civile et judiciaire de la colonie était dominée par quelques familles. Elles n'appartenaient pas à une aristocratie militaire mais formaient une élite seigneuriale, militaire, commerciale, administrative. ${ }^{14}$

Selon Nish, cette élite se compare bien avec la classe dominante des colonies britanniques, ce qui relativise le poids des indicateurs idéologiques dans l'explication historique puisqu'avec ou sans éthique protestante/mentalité capitaliste on retrouve des structures de classes sensiblement identiques. Nish utilise trois critères: monopole du commerce, monopole du pouvoir politique et cohésion sociale pour montrer que dans l'ensemble les structures économiques de la Nouvelle-France et de la Nouvelle-Angleterre sont comparables, que par conséquent les différences d'idéologies ne sont pas significatives et qu'en définitive l'existence d'une bourgeoisie en Nouvelle-France est bien fondée, bourgeoisie en train de se constituer dans le cadre de la transition entre le féodalisme et le capitalisme. En fait, comme on peut le constater, le critère qui est sous-jacent à toutes ces analy-

13 Cameron Nish, Les bourgeois gentilshommes (Montréal, Fides, 1968), 23. Ibid., 156. 
ses, c'est la capacité d'accumuler. Pour Hamelin et Ouellet, l'activité économique de la colonie a contribué à l'enrichissement de la métropole et non pas à la prospérité du pays et de sa bourgeoisie alors que Michel Brunet et $\mathrm{C}$. Nish tentent de démontrer le contraire. En réalité, l'enjeu de ce débat est la lutte pour la légitimité historique entre deux fractions de la classe dominante canadienne-française, l'interprétation d'Hamelin et Ouellet cautionnant la pratique et l'idéologie de la collaboration de l'élite canadienne-française à la colonisation britannique, celle de Brunet, Nish et autres mettant à l'avant-plan les effets débilitants de la Conquête et de la domination britannique, et justifiant ainsi la pratique et l'idéologie autonomiste visant la reconstitution d'une bourgeoisie nationale dans le cadre et par un État indépendant.

Dans cette historiographie, on a l'impression qu'il n'y a qu'un seul acteur sur la scène de l'histoire de sorte qu'on pourrait dire que jusqu'à présent l'histoire du Québec n'a été que l'histoire de sa classe dominante et des luttes entre ses fractions. L'histoire des producteurs de la richesse relève de l'anecdote et du complément.

D'un côté, on a cherché à montrer qu'il n'y avait pas de servage puisque le capitalisme se développait et de l'autre qu'il n'y avait pas de prolétariat puisqu'on était en régime féodal. À la limite, si on mettait bout à bout ces diverses interprétations, on se retrouverait avec une société sans classe, sans bourgeoisie, sans noblesse, sans serfs et sans prolétariat. Paradoxe de l'histoire ou lacune des historiens? Nous penchons plutôt pour la seconde hypothèse... Et pour combler cette lacune, nous pensons que le concept de MPPP pourrait être utile car il est, à notre avis, mieux adapté pour décrire une situation de transition. Il a aussi l'avantage de prendre en considération un facteur-clé pour comprendre la nature d'une formation sociale, le processus de la division du travail et le rapport aux moyens de production. Notre intention, ici, n'est pas de reprendre l'analyse de la Nouvelle-France de façon systématique, nous nous contenterons de suggérer quelques éléments qui semblent démontrer la fécondité du concept de MPPP pour la compréhension de la formation sociale de la Nouvelle-France et des effets de la Conquête, en espérant que cette exploration ouvrira des pistes de recherches.

D'une manière générale, on peut admettre que l'état de développement des rapports de production et des rapports sociaux en Nouvelle-France dépend de l'état de ces mêmes rapports en métropole et que cette période peut être caractérisée comme étant la 
phase pré-industrielle du capitalisme, c'est-à-dire une phase où l'accumulation se fait beaucoup plus à partir du commerce que de la production.

Toutefois, la reproduction ne peut être identique car d'une part la colonisation répond à des exigences spécifiques propres à certains secteurs de la structure sociale métropolitaine et rencontre aussi des conditions spécifiques à la périphérie qui nécessitent certains ajustements. Le système socio-économique de la Nouvelle-France ne se comprend qu'en tant que projection de la société française et qu'en tant que déviance par rapport à ce modèle. L'interprétation qui postule un transfert intégral dans le Nouveau Monde de l'ordre social féodal de l'Ancien Monde est insoutenable. La perspective suivante nous apparaît plus juste: l'asymétrie synchronique de l'organisation économique et sociale de la colonie par rapport à celle de la métropole est fonction des nécessités économiques et politiques de la métropole médiatisées par les conditions internes à la colonie.

«De ses propres mains, écrit Sigmud Diamond, la France a créé au Canada une base sociale pour la désobéissance, un cadre social dans lequel les déviations devenaient l'unique moyen de survivance ou de faire quelque profit. " ${ }^{15}$ Cette déviance se produit non seulement par rapport au féodalisme, mais aussi par rapport au capitalisme tel qu'il se développe en Europe par le processus de prolétarisation car le capitalisme, avant d'être un système d'accumulation, est un système d'exploitation des travailleurs produisant la plusvalue. Donc pour qu'il y ait capitalisme il ne suffit pas qu'il y ait une bourgeoisie marchande, il faut qu'il y ait aussi un prolétariat, c'està-dire une séparation entre le producteur et ses instruments de production. Cette séparation est la condition indispensable au développement d'une économie de marché et elle n'a pu être réalisée en Nouvelle-France. On ne peut, écrit Larry MacDonald, considérer les Indiens comme des prolétaires: "The French were not purchasing native labour-power, they must be understood as purchasing the product of native labour, this is furs.» ${ }^{16}$ L'existence des Indiens dépendait de la forêt et non pas des Français qui étaient des marchands et pas ou très peu des employeurs. On a donc ici un exemple où la pénétration colonisatrice ne détruit pas le mode de production autochtone, mais se fait en cœxistence avec ce dernier avec lequel le

15 Sigmund Diamond, "Le Canada français au XVIIième siècle: une société préfabriquée», Annales (mars-avril 1961): 353.

16 Larry MacDonald, op.cit., 130. 
mode de production dominant entretient des rapports d'échange et non de production. Il en ira autrement au $\mathrm{XIX}^{\mathrm{e}}$ siècle.

Ce sont les conditions mêmes de l'exploitation coloniale qui imposèrent la déviation dans l'articulation des modes de production de la formation sociale coloniale, c'est-à-dire qui permirent le développement du MPPP en Nouvelle-France.

Dans son processus d'expansion, le capitalisme commercial s'est servi du MPPP pour faire face aux conditions spécifiques des territoires conquis et à la situation particulière de l'échange entre la métropole et la colonie, c'est-à-dire qu'à la périphérie pour assurer la reproduction de la force d'exploitation ou autrement dit pour assurer la subsistance des colonisateurs et réduire les frais d'exploitation de la traite des fourrures, on n'a pu effectuer la séparation du producteur de ses moyens de production car un certain degré d'autosuffisance était nécessaire au développement même du capitalisme. La non-spécialisation fut alors une condition de survie de la colonie, de même que le maintien d'une économie agraire indépendante du mode de production capitaliste.

En effet, cette politique coloniale est soumise à des impératifs contradictoires. Seule une colonie d'exploitation était rentable, mais le maintien du monopole commercial était impossible sans le peuplement et une colonie de peuplement diminuait sensiblement le taux de profit. Le peuplement de l'Amérique par des paysans-soldats s'imposait pour le contrôle du monopole de la traite avec les Indiens, permettant un soutien militaire aux Montagnais, Algonquins et Hurons dans leurs luttes contre les Iroquois. Par cette politique d'alliance, il s'agissait d'étendre la zone d'influence française et d'enrayer les perturbations de la traite des fourrures. L'autre raison pourrait être la concurrence avec l'Angleterre pour le contrôle de ces nouvelles sources de richesses. À cet égard, de simples postes de traite étaient insuffisants pour coloniser les Indiens et résister aux prétentions monopolistes anglaises. On peut donc dire que la stratégie coloniale française est soumise à la dialectique exploitation/rentabilité/peuplement qui, dans cette perspective, n'est qu'un alibi, qu'un soutien à la politique d'exploitation commerciale qui est l'objectif primordial.

Afin d'amoindrir les coûts d'opération de la colonie, l'État absolutiste tenta de diversifier la production et de développer l'autosuffisance. Mais pour réaliser ce programme, l'État fut forcé de ga- 
rantir aux habitants le libre accès aux moyens de production et de subsistance:

in New France, the necessity of establishing production, in the absence of economic or physical compulsion on labour to produce more than was necessary for its own subsistance, required the establishment and maintenance of conditions guaranteeing the freedom of labour from just such compulsion. ${ }^{17}$

Sigmund Diamond ajoute à ce sujet:

Au Canada français... l'utilisation du travail forcé et des Indiens autochtones se solda par un échec, et il fallut recourir aux travailleurs volontaires. Pour les recruter et les amener au travail, il fallait user de la persuasion et de l'intérêt. Les conccssions faites à la main-d'oeuvre française furent si substantielles qu'elles la mirent dans une position totalement différente non seulement de celle qu'elle occupait dans le Vieux Monde, mais, non moins, de celle que les planificateurs entendaient lui faire occuper... Pour l'Amérique du Nord, la nécessité de recruter une main-d'oeuvre fut la mère de toutes les libertés. ${ }^{18}$

Il n'y a pas eu en Nouvelle-France de processus de dépossession des moyens de production comme ce fut le cas en Europe dans la transition du féodalisme au capitalisme. Il n'y a pas eu dépossession terrienne créant une force de travail libre, élément essentiel au développement du capitalisme; au contraire, les immigrants qui arrivaient au Canada échappaient justement à ce processus de prolétarisation, de séparation entre le travailleur et ses moyens de production. Mais il n'y a pas non plus de servage en Nouvelle-France. Le paysan est propriétaire de son lopin de terre et il est le seul maître de son travail et de ses produits. L'accès à la terre était libre non pas tellement en raison des vastes espaces, mais surtout en vertu des concessions des administrateurs coloniaux. Il faut aussi noter que "la distribution du sol rural est faite sous le signe de l'égalité » ${ }^{19}$ et qu'il y a absence de morcellement de la propriété.

À l'inverse du paysan français ou anglais, le paysan canadien n'était pas forcé de vendre sa force de travail pour vivre. Il était un travailleur indépendant assurant par sa production son autosubsistance et celle de sa famille. D'ailleurs, cette différence de situation objective se traduisait au niveau de la conscience car les premiers

17 Ibid., 124.

18 Sigmund Diamond, op. cit.: 338 et 354.

19 Louise Dechêne, op.cit., 265. 
Canadiens voulant se démarquer des censitaires tenaient à ce qu'on les appelle «habitant», qui signifiait homme libre, possesseur du sol, et fixé en permanence dans la colonie.

Même si au niveau des rapports sociaux, le seigneur jouit d'un certain prestige et d'une certaine prééminence, au niveau des rapports de production, paysans et seigneurs ne sont pas dans un rapport de dépendance. Le seigneur assure son existence plus par ses activités commerciales en tant que négociant - ce qu'il peut faire sans déroger - que de la rente ou de l'appropriation de la production des habitants. Il lui répugnera règle générale de développer sa seigneurie et préférera s'occuper de la traite des fourrures laissant ainsi aux paysans une grande marge d'autonomie. Les seigneurs au Canada ne sont pas pour la plupart d'origine noble et leur statut socio-économique n'est pas lié à leur titre de noblesse. Les seigneuries étaient concédées à une bourgeoisie en formation composée à la fois de propriétaires terriens, de marchands, de militaires et d'administrateurs, le même individu pratiquant souvent ces différentes activités. «Il se mariaient entre eux; ils vivaient non pas humblement sur une ferme mais bien dans les villes de la colonie.» ${ }^{20}$

Cette particularité infrastructurelle s'est aussi traduite au niveau des rapports sociaux et des institutions qui les incarnent. Rappelons brièvement à cet égard les aspects originaux du régime seigneurial canadien que Jean-Pierre Wallot résume ainsi:

le régime seigneurial a engendré un nouveau type social dont il consolide les intérêts: l'habitant indépendant, exempt d'impôt personnel, propriétaire de sa terre, très mobile à cause de la traite et de l'abondance des terres, libéré des corvées seigneuriales et sur le même pied que le seigneur vis-à-vis les pratiques communautaires. ${ }^{21}$

À notre avis, le régime seigneurial canadien n'est qu'une pellicule féodaliste qui recouvre en réalité le MPPP et se différencie fondamentalement du régime seigneurial français par la relative désuétude de certains droits seigneuriaux, par la forme originale de la structure familiale et du système d'héritage, par la souplesse des barrières sociales, par le désintérêt relatif des seigneurs envers les revenus de leur seigneurie et enfin par l'absence des attributs de la souveraineté légale et politique pour les seigneurs ${ }^{22}$.

\footnotetext{
20 Cameron Nish, op.cit., 115.

21 Jean-Pierre Wallot, "Le régime seigneurial et son abolition au Canada", Canadian Historical Review, L, 4 (décembre 1969): 375.

22 Sigmund Diamond, op. cit. : 323-324.
} 
D'ailleurs, en Nouvelle-France, il n'y avait pratiquement pas d'expropriation de la production. La dîme représentait $4 \%$ de la production et la rente seigneuriale - lorsqu'elle était perçue - $11 \%$, alors que ces extorsions atteignaient $30 \%$ pour les paysans français. L'habitant était aussi libre d'étendre sa production pour assurer son autosubsistance ce qui lui permettait d'éviter le marché du travail de sorte que son niveau de vie se compare bien à celui des paysans indépendants en France. L'immigration en Nouvelle-France était une garantie de mobilité sociale.

Lorsque les seigneurs ou le clergé exigeaient le paiement des rentes et dîmes ou lorsqu'ils voulaient les élever, les habitants résistaient et refusaient farouchement de payer: "Plusieurs particuliers, écrivait Duchesneau en 1677, par une désobéissance manifeste (...) et par un mépris pour l'Église, non seulement refusent de payer les dixmes, mais mesme s'emportent jusques à la viollance.» ${ }^{23} \AA \grave{A}$ Longueuil, il y a eu un soulèvement populaire armé qui dura deux jours pour contester les corvées d'État. Les habitants ne s'écrasent pas devant l'autorité royale, ils ont plutôt tendance à être indisciplinés. À plusieurs reprises le peuple imposera sa volonté et obligera l'administration à reculer. Il semble bien que l'individualisme et l'esprit d'indépendance soient deux des éléments dominants de la mentalité des Canadiens sous le régime français. Cet esprit de liberté était accompagné, selon certains commentateurs, d'un égalitarisme social: «Ici tout le monde est monsieur ou madame, le paysan aussi bien que le gentilhomme, la paysanne comme la plus grande dame.» Cette affirmation quoiqu'empreinte d'idyllisme, révèle le caractère particulier des rapports sociaux en Nouvelle-France.

Enfin, dans le système féodal classique par la maîtrise de la justice, les seigneurs avaient le pouvoir d'exiger l'obéissance: "Or, aux mains des seigneurs, écrit Marc Bloch, l'exercice presque sans restriction des droits de justice, a mis une arme d'exploitation économique infiniment redoutable... Le droit de juger avait été le plus sûr appui du droit d'ordonner.» ${ }^{24}$ Au Canada, une telle institution de la justice n'existait pas. Il était impossible à un seigneur de se soumettre un habitant. C'étaient les habitants et non les seigneurs que l'on désignait comme capitaine de milice: poste qui impliquait l'exercice de l'autorité civile autant que militaire.

23 Cité par Sigmund Diamond, ibid.: 350.

24 Marc Bloch, Caractères originaux de l'histoire rurale française (Paris, Colin, 1952), 82 et 84 . 
Certes, au niveau institutionnel, la Nouvelle-France vit dans le cadre de l'absolutisme, mais les gouverneurs éprouvent beaucoup de difficultés à faire respecter la loi. L'insubordination et la désobéissance étaient fort répandues dans la colonie puisque le pouvoir de répression de l'appareil d'État était faible en raison des distances, du faible réseau de communications et de l'éparpillement des habitants ${ }^{25}$. Il est donc hasardeux, comme le font certains, de suggérer qu'à cause de son caractère absolutiste, le pouvoir en Nouvelle-France était tyrannique, autoritaire et paternaliste. En raison des conditions du milieu et de la spécificité de la situation coloniale, l'absolutisme ne s'est pas traduit par un asservissement collectif et un despotisme. Il faut à cet égard se méfier de la propagande intoxicante des conquérants britanniques infiltrée dans la pensée de certains historiens qui prétendent avec Lord Durham que «les institutions françaises à l'époque de la colonisation du Canada étaient peut-être plus que celles de toute autre nation européenne conçues en vue de réprimer l'intelligence et la liberté des masses populaires ${ }^{26}$. S'il faut se méfier de l'idéologie du colonisateur dans l'interprétation de l'histoire, il ne faut pas pour autant tomber dans l'idéalisme et faire de la Nouvelle-France l'Icarie réalisée. Il faut tenir compte des conditions matérielles, comprendre comment elles interviennent au niveau des superstructures et ainsi produire des hypothèses plausibles qui tiennent compte des spécificités et des possibilités de distorsions, de décalages.

L'habitant de la Nouvelle-France produisait donc son existence dans une structure de type féodaliste empruntée à la structure métropolitaine mais qui allait en colonie servir d'autres impératifs (développement du capitalisme dans sa phase commerciale) et prendre un aspect spécifique: absence de servage, non-séparation entre le travailleur et ses moyens de production, indépendance de l'habitant par rapport au seigneur, non-spécialisation des tâches, quasi autosuffisance (sauf pour les produits de luxe) et caractère secondaire de la production pour le marché. À cet égard, certaines observations faites par Louise Dechêne confirment la pertinence de cette dernière proposition. Se basant sur une analyse de la comptabilité d'Alexis Monière, elle soutient que les rapports d'échange entre marchands et

25 R.-L. Séguin, «L'esprit d'insubordination en Nouvelle-France et au Québec au XVIIième et XVIIIième siècles", L'Académie des sciences d'outre-mer, XXXIII, 4 (1973). 1944), 125.

26 G. Frégault, La civilisation de la Nouvelle-France (Montréal, Ed. Pascal 
habitants sont secondaires par rapport au chiffre d'affaires global des premiers, que le marchand ne réalise pas ou peu de profits dans cet échange, ensuite que la production artisanale échappe totalement au contrôle du marchand, et enfin, qu'il y a absence de liaison entre le capital commercial et la production locale de sorte qu'on peut dire que pour ce secteur d'activités, la valeur d'usage prime sur la valeur d'échange et que la production se développe en retrait de l'économie de marché ${ }^{27}$. Ces divers attributs manifestent bien la présence sous pellicule féodaliste du MPPP. Il faut souligner que ce modèle économique n'est pas en concurrence avec le développement du capitalisme, il lui sert plutôt d'appui et facilite l'accumulation primitive du capital. Il absorbe environ $75 \%$ de la main-d'oeuvre de la colonie, l'autre portion tirant sa subsistance des activités commerciales et des fonctions administratives ou de leur combinaison. On peut donc dire que dans l'articulation des modes de production en NouvelleFrance, le féodalisme n'est que symbolique, le capitalisme a un rôle prédominant et donne un sens à l'existence de la colonie, appuyé en cela par le MPPP qui fournit la basc existenticlle de l'activité commerciale en reproduisant la capacité d'échanger, de faire le commerce des fourrures.

Mais le développement économique sous l'égide du capitalisme, en raison de la nécessité de la concentration des capitaux et de la généralisation de la marchandise conduit inéluctablement au dépérissement du MPPP et à la constitution d'un prolétariat. Le petit producteur indépendant n'a pas d'avenir sur le plan historique, car sa sphère d'activités tôt ou tard sera dominée par la logique de la marchandise. Le MPPP, privé d'antagonismes de classes, n'a pas les éléments nécessaires pour être le moteur d'une société. Son existence est toujours conditionnée par la dominance d'un autre type de rapports de l'homme à la nature et avec ses semblables, en l'occurrence le capitalisme dans sa phase commerciale en ce qui concerne la Nouvelle-France. Cependant force est de constater que ce processus de dissolution progressive a été bloqué ou retardé momentanément au Canada. D'élément dominé qu'il était sous le régime fran-

27 Louise Dechêne, op.cit., 186-187, 230. Cette dimension importante du MPPP est aussi attestée par un commentaire de l'ingénieur du roi Franquet qui écrit: «L'on doit juger que l'habitant des campagnes est trop à son aise, et que ce serait faire le bien de la colonie en général de la charger un peu pour l'obliger comme on l'a dit cy-devant à venir en ville y apporter les denrées et s'y procurer en marchandises ce dont il peut avoir besoin." Cité par R.-L. Séguin, La civilisation traditionnelle de l'habitant aux 17,e et $18^{e}$ siècles (Montréal, Fides, 1967), 53. 
çais, le MPPP deviendra après la Conquête dominant pour la structure sociale canadienne-française, permettant par le fait même la domination de la petite bourgeoisie et de son idéologie.

\section{Conclusion}

Cette brève prospection nous montre que le concept de MPPP est applicable à la Nouvelle-France et qu'il peut être fécond pour en comprendre le développement. Il permet, à notre avis, d'approfondir la connaissance des fondements économiques de cette formation sociale, en mettant en lumière la complexité du processus de transition du féodalisme au capitalisme et la spécificité du processus de colonisation. Il donne une autre perspective à la compréhension des effets de la Conquête relativisant la thèse de l'absence de changements dans la structure économique interne de la colonie. À cet égard, la théorie de la destruction de la bourgeoisie coloniale canadienne et son remplacement par une bourgeoisie anglaise, à elle seule, est insatisfaisante pour expliquer la nature de la formation sociale canadienne après 1760 . Elle a une vertu beaucoup plus descriptive qu'explicative. Certes, le capitalisme continue de se développer à partir de l'exploitation du même produit de base et il y a formation d'une double structure de classes à superposition nationale, mais quels sont les modes de production sous-jacents à ces structures de classes? L'historiographie actuelle semble considérer qu'il n'y a que le mode de production capitaliste à la base des deux structures sociales en présence et aboutit à la conclusion qu'en définitive il y a une nation bourgeoise et une nation prolétaire dans laquelle on retrouve une classe dominante inféodée à la nation bourgeoise et des classes dominées. N'y aurait-il pas lieu de repenser le fondement économique de la structure de classes dominée et pour ce faire d'utiliser le concept de MPPP? Cette hypothèse nous permettrait de mieux comprendre le poids de la petite bourgeoisie dans le développement historique de la société québécoise. Entre autres, elle serait, pensons-nous, pertinente pour donner une appréciation plus juste de l'idéologie de Papineau et des Patriotes que celle proposée par F. Ouellet et F. Dumont qui attribuent à Papineau une mentalité féodale en raison de sa défense du régime seigneurial ${ }^{28}$.

28 Voir Fernand Dumont, Idéologies au Canada français (1850-1900) (Québec, Presses de l'Université Laval, 1971), 67-68. 
L'histoire du Québec gagnerait à être étudiée à travers la problématique de la transition et de l'articulation des modes de production. En somme, le débat reste ouvert. 